\title{
Hospitalist Perspective of Interactions with Medicine Subspecialty Consult Services
}

\author{
Traci N. Adams, MD*, Joanna Bonsall, MD², Daniel Hunt, MD², Alberto Puig, MD³, Jeremy B. Richards, MD4, Liyang Yu, MS ${ }^{5}$
} Jakob I. McSparron, $\mathrm{MD}^{6}$, Nainesh Shah, MD , Jonathan Weissler, MD¹, Eli M. Miloslavsky, MD ${ }^{8}$

\begin{abstract}
${ }^{1}$ Division of Pulmonary and Critical Care Medicine, University of Texas Southwestern Medical Center, Dallas, Texas; ${ }^{2}$ Department of Medicine, Emory University, Atlanta, Georgia; ${ }^{3}$ Department of Medicine, Massachusetts General Hospital, Boston, Massachusetts; ${ }^{4}$ Division of Pulmonary, Critical Care, and Sleep Medicine, Medical University of South Carolina, Charleston, South Carolina; ${ }^{5}$ Division of Internal Medicine, Massachusetts General Hospital, Boston, Massachusetts; ${ }^{6}$ Division of Pulmonary, Critical Care, and Sleep Medicine, Beth Israel Deaconess Medical Center, Boston, Massachusetts; 'Department of Medicine, University of Texas Southwestern Medical Center, Dallas, Texas; ${ }^{8}$ Division of Rheumatology, Allergy, and Immunology, Massachusetts General Hospital, Boston, Massachusetts.
\end{abstract}

BACKGROUND: Medicine subspecialty consultation is becoming increasingly important in inpatient medicine.

OBJECTIVE: We conducted a survey study in which we examined hospitalist practices and attitudes regarding medicine subspecialty consultation.

DESIGN AND SETTING: The survey instrument was developed by the authors based on prior literature and administered online anonymously to hospitalists at 4 academic medical centers in the United States.

MEASUREMENTS: The survey evaluated 4 domains: (1) current consultation practices, (2) preferences regarding consultation, (3) barriers to and facilitating factors of effective consultation, and (4) a comparison between hospitalist-fellow and hospitalist-subspecialty attending interactions.

RESULTS: One hundred twenty-two of 261 hospitalists (46.7\%) responded. The majority of hospitalists interacted with fellows during consultation. Of those, $90.9 \%$ reported that in-person communication occurred during less than half of consultations, and $64.4 \%$ perceived pushback at least "sometimes" in their consult interactions. Participants viewed consultation as an important learning experience, preferred direct communication with the consulting service, and were interested in more teaching during consultation. The survey identified a number of barriers to and facilitating factors of an effective hospitalist-consultant interaction, which impacted both hospitalist learning and patient care. Hospitalists reported more positive experiences when interacting with subspecialty attendings compared to fellows with regard to multiple aspects of the consultation.

CONCLUSION: The hospitalist-consultant interaction is viewed as important for both hospitalist learning and patient care. Multiple barriers and facilitating factors impact the interaction, many of which are amenable to intervention. Journal of Hospital Medicine 2018;13:318323. Published online first November 22, 2017. (02018 Society of Hospital Medicine ospitalist physicians care for an increasing proportion of general medicine inpatients and request a significant share of all subspecialty consultations. ${ }^{1}$ Subspecialty consultation in inpatient care is increasing $_{1}{ }^{2,3}$ and effective hospitalist-consulting service interactions may affect team communication, patient care, and hospitalist learning. Therefore, enhancing hospitalist-consulting service interactions may have a broad-reaching, positive impact. Researchers in previous studies have explored resident-fellow consult interactions in the inpatient and emergency depart-

\footnotetext{
*Address for correspondence: Traci N. Adams, MD, UT Southwestern Medica Center, 5323 Harry Hines Blvd, Dallas, TX 75219; Telephone: 832-428-8135; Fax 214-645-6272; E-mail: tnfadams@gmail.com

Additional Supporting Information may be found in the online version of this article.
}

Received: June 1, 2017; Revised: August 16, 2017;

Accepted: August 18, 2017

2018 Society of Hospital Medicine DOI 10.12788/jhm.2882 ment settings as well as attending-to-attending consultation in the outpatient setting. ${ }^{4-7}$ However, to our knowledge, hospitalist-consulting team interactions have not been previously described. In academic medical centers, hospitalists are attending physicians who interact with both fellows (supervised by attending consultants) and directly with subspecialty attendings. Therefore, the exploration of the hospitalist-consultant interaction requires an evaluation of hospitalist-fellow and hospitalist-subspecialty attending interactions. The hospitalist-fellow interaction in particular is unique because it represents an unusual dynamic, in which an attending physician is primarily communicating with a trainee when requesting assistance with patient care. ${ }^{8}$ In order to explore hospitalistconsultant interactions (herein, the term "consultant" includes both fellow and attending consultants), we conducted a survey study in which we examine hospitalist practices and attitudes regarding consultation, with a specific focus on hospitalist consultation with internal medicine subspecialty consult services. In addition, we compared fellow-hospitalist and attending- 
hospitalist interactions and explored barriers to and facilitating factors of an effective hospitalist-consultant relationship.

\section{METHODS}

\section{Survey Development}

The survey instrument was developed by the authors based on findings of prior studies in which researchers examined consultation. ${ }^{2-6,9-16}$ The survey contained 31 questions (supplementary Appendix A) and evaluated 4 domains of the use of medical subspecialty consultation in direct patient care: (1) current consultation practices, (2) preferences regarding consultants, (3) barriers to and facilitating factors of effective consultation (both with respect to hospitalist learning and patient care), and (4) a comparison between hospitalist-fellow and hospitalist-subspecialty attending interactions. An evaluation of current consultation practices included a focus on communication methods (eg, in person, over the phone, through paging, or notes) because these have been found to be important during consultation. $5,6,9,15,16$ In order to explore hospitalist preferences regarding consult interactions and investigate perceptions of barriers to and facilitating factors of effective consultation, questions were developed based on previous literature, including our qualitative work examining resident-fellow interactions during consultation. ${ }^{4-6,9,12}$ We compared hospitalist consultation experiences among attending and fellow consultants because the interaction in which an attending hospitalist physician is primarily communicating with a trainee may differ from a consultation between a hospitalist attending and a subspecialty attending. ${ }^{8}$ Participants were asked to exclude their experiences when working on teaching services, during which students or housestaff often interact with consultants. The survey was cognitively tested with both hospitalist and non-hospitalist attending physicians not participating in the study and was revised by the authors using an iterative approach.

\section{Study Participants}

Hospitalist attending physicians at University of Texas Southwestern (UTSW) Medical Center, Emory University School of Medicine, Massachusetts General Hospital (MGH), and the Medical University of South Carolina (MUSC) were eligible to participate in the study. Consult team structures at each institution were composed of either a subspecialist-attending-only or a fellow-and-subspecialty-attending team. Fellows at all institutions are supervised by a subspecialty attending when performing consultations. Respondents who self-identified as nurse practitioners or physician assistants were excluded from the analysis. Hospitalists employed by the Veterans Affairs hospital system were also excluded. The study was approved by the institutional review boards of UTSW, Emory, MUSC, and $\mathrm{MGH}$.

The survey was anonymous and administered to all hospitalists at participating institutions via a web-based survey tool (Qualtrics, Provo, UT). Participants were eligible to enter a raffle for a $\$ 500$ gift card, and completion of the survey was not required for entry into the raffle.
TABLE 1. Participant Baseline Data

\begin{tabular}{|c|c|}
\hline Characteristics & $\mathrm{N}(\%)$ \\
\hline \multicolumn{2}{|l|}{ Gender } \\
\hline Male & $63(51.6)$ \\
\hline Female & $59(48.4)$ \\
\hline Age (mean +/- SD) & $37.7+/-7.9$ \\
\hline \multicolumn{2}{|l|}{ Primary practice site } \\
\hline Academic medical center & $105(86.1)$ \\
\hline Community nonteaching hospital & $2(1.6)$ \\
\hline Community teaching hospital & $14(11.5)$ \\
\hline Years worked as a hospitalist (mean +/- SD) & $5.6+/-5.0$ \\
\hline Years worked in current institution (mean +/- SD) & $3.6+/-2.9$ \\
\hline Percentage of daytime shifts (mean +/- SD) & $74.1+/-35.1$ \\
\hline Percentage of time on teaching services (mean $+/$ - SD) & $19.2+/-25.1$ \\
\hline Percentage of time on direct patient care (mean +/- SD) & $70.5+/-34.0$ \\
\hline \multicolumn{2}{|l|}{ Use of consult services over time } \\
\hline Increased a lot & $9(7.4)$ \\
\hline Increased a little & $38(31.1)$ \\
\hline No change & $38(31.1)$ \\
\hline Decreased a little & $30(24.6)$ \\
\hline Decreased a lot & $7(5.7)$ \\
\hline \multicolumn{2}{|l|}{ Total consults per shift } \\
\hline $0-1$ & $48(39.3)$ \\
\hline $2-3$ & $62(50.8)$ \\
\hline $4-5$ & $8(6.6)$ \\
\hline$>5$ & $2(1.6)$ \\
\hline Medical subspecialty consults per shift (mean +/- SD) & $2.9+/-2.4$ \\
\hline \multicolumn{2}{|l|}{ Most common reason for requesting consultation } \\
\hline Assistance with diagnosis & $26(21.3)$ \\
\hline Assistance with treatment & $49(40.2)$ \\
\hline Request a procedure & $22(18.0)$ \\
\hline Patient request & $4(3.3)$ \\
\hline Discharge planning & $0(0)$ \\
\hline
\end{tabular}

\section{Statistics}

Results were summarized using the mean with standard deviation for continuous variables and the frequency with percentage for categorical variables after excluding missing values. All analyses were conducted using SAS version 9.4 (SAS Institute, Cary, NC). A 2-sided $P$ value of $\leq .05$ was considered statistically significant.

\section{RESULTS}

Of a possible 261 respondents, 122 (46.7\%) participated in the survey. Missing values for survey responses ranged from $0 \%$ to $21.3 \%$, with a mean of $15.2 \%$. Demographic characteristics are shown in Table 1. Respondents had a mean age of 37.7 years and had worked as attending hospitalists for an average of 5.6 years. The majority of respondents $(86.1 \%)$ practiced in aca- 
TABLE 2. Hospitalist Consultation Practices

\begin{tabular}{|c|c|c|c|c|c|c|}
\hline Practices & $\mathrm{N}$ & Never & Sometimes & $\begin{array}{l}\text { About Half } \\
\text { the Time }\end{array}$ & $\begin{array}{l}\text { Most of } \\
\text { the Time }\end{array}$ & Always \\
\hline Consults performed by fellow with attending supervision & 102 & $4(3.9 \%)$ & $5(4.9 \%)$ & $10(9.8 \%)$ & $53(52 \%)$ & $30(29.4 \%)$ \\
\hline Hospitalist speaks with consultant to request consult in person & 97 & $25(25.8 \%)$ & $59(60.8 \%)$ & $10(10.3 \%)$ & $3(3.1 \%)$ & $0(0 \%)$ \\
\hline Hospitalist speaks with consultant to request consult over the phone & 101 & $3(3 \%)$ & $12(11.9 \%)$ & $9(8.9 \%)$ & $46(45.5 \%)$ & $31(30.7 \%)$ \\
\hline Hospitalist speaks with consultant to request consult by page only & 96 & $46(47.9 \%)$ & $34(35.4 \%)$ & $3(3.1 \%)$ & $8(8.3 \%)$ & $5(5.2 \%)$ \\
\hline Consultant communicates with hospitalist after evaluating patient in person & 99 & $24(24.2 \%)$ & $66(66.7 \%)$ & $9(9.1 \%)$ & $0(\%)$ & $0(\%)$ \\
\hline Consultant communicates with hospitalist after evaluating patient over the phone & 103 & $2(1.9 \%)$ & $38(36.9 \%)$ & $25(24.3 \%)$ & $34(33 \%)$ & $4(3.9 \%)$ \\
\hline Consultant communicates with hospitalist after evaluating patient by page only & 100 & $14(14 \%)$ & $71(71 \%)$ & $10(10 \%)$ & $5(5 \%)$ & $0(0 \%)$ \\
\hline Percentage of consults where hospitalists learned from interactions with consultant & 102 & $1(1 \%)$ & $43(42.2 \%)$ & $37(36.3 \%)$ & $17(16.7 \%)$ & $4(3.9 \%)$ \\
\hline
\end{tabular}

demic medical centers, with the remaining working in satellite community hospitals. Respondents reported working daytime shifts $74.1 \%$ of the time on average and being on inpatient, direct-care services without house-staff $70.5 \%$ of the time.

\section{Current Consultation Practices}

Current consultation practices and descriptions of hospitalistconsultant communication are shown in Table 2. Forty percent of respondents requested $0-1$ consults per day, while $51.7 \%$ requested 2-3 per day. The most common reasons for requesting a consultation were assistance with treatment (48.5\%), assistance with diagnosis (25.7\%), and request for a procedure (21.8\%). When asked whether the frequency of consultation is changing, slightly more hospitalists felt that their personal use of consultation was increasing as compared to those who felt that it was decreasing (38.5\% vs $30.3 \%$, respectively).

An exploration of communication practices during consultation revealed that hospitalists most often interacted with fellows rather than attending physicians (81.4\%). However, even when a fellow performs a consult and communicates with a hospitalist, a subspecialty attending is involved in the care of the patient, although he or she may not communicate directly with the hospitalist. Respondents indicated that they most often communicated a consult request to the consultant by phone (76.2\%). Pushback from consultants (defined as perceived reluctance or resistance to perform the consult for any reason) was perceived as common, with $64.4 \%$ of hospitalists indicating that they experience pushback at least "sometimes" (3 on a 5-point Likert scale) and $22.1 \%$ reporting that pushback was "frequent" or occurred "most of the time". Follow-up interactions (defined as communication of recommendations after the consultant evaluated the patient) infrequently occurred through in-person communication, with $90.9 \%$ reporting that this occurred in less than half of consultations. Communication by phone was most common, with $61.2 \%$ reporting that it occurred at least half the time, and $86 \%$ of respondents reported that communication by paging only occurred at least "sometimes". Consultation was commonly seen as a valuable educational experience, with $56.9 \%$ of hospitalists indicating that they learned from at least half of consults.

\section{Hospitalist Preferences}

Eighty-six percent of respondents agreed that consultants should be required to communicate their recommendations either in person or over the phone. Eighty-three percent of hospitalists agreed that they would like to receive more teaching from the consulting services, and $74.0 \%$ agreed that consultants should attempt to teach hospitalists during consult interactions regardless of whether the hospitalist initiates the teaching-learning interaction.

\section{Barriers to and Facilitating Factors of Effective Con- sultation}

Participants reported that multiple factors affected patient care and their own learning during inpatient consultation (Figure 1). Consultant pushback, high hospitalist clinical workload, a perception that consultants had limited time, and minimal in-person interactions were all seen as factors that negatively affected the consult interaction. These generally affected both learning and patient care. Conversely, working on an interesting clinical case, more hospitalist free time, positive interaction with the consultant, and having previously worked with the consultant positively affected both learning and patient care (Figure 1).

\section{Fellow Versus Attending Interactions}

Respondents indicated that interacting directly with the consult attending was superior to hospitalist-fellow interactions in all aspects of care but particularly with respect to pushback, confidence in recommendations, professionalism, and hospitalist learning (Figure 2).

\section{DISCUSSION}

To our knowledge, this is the first study to describe hospitalist attending practices, attitudes, and perceptions of internal medicine subspecialty consultation. Our findings, which focus 


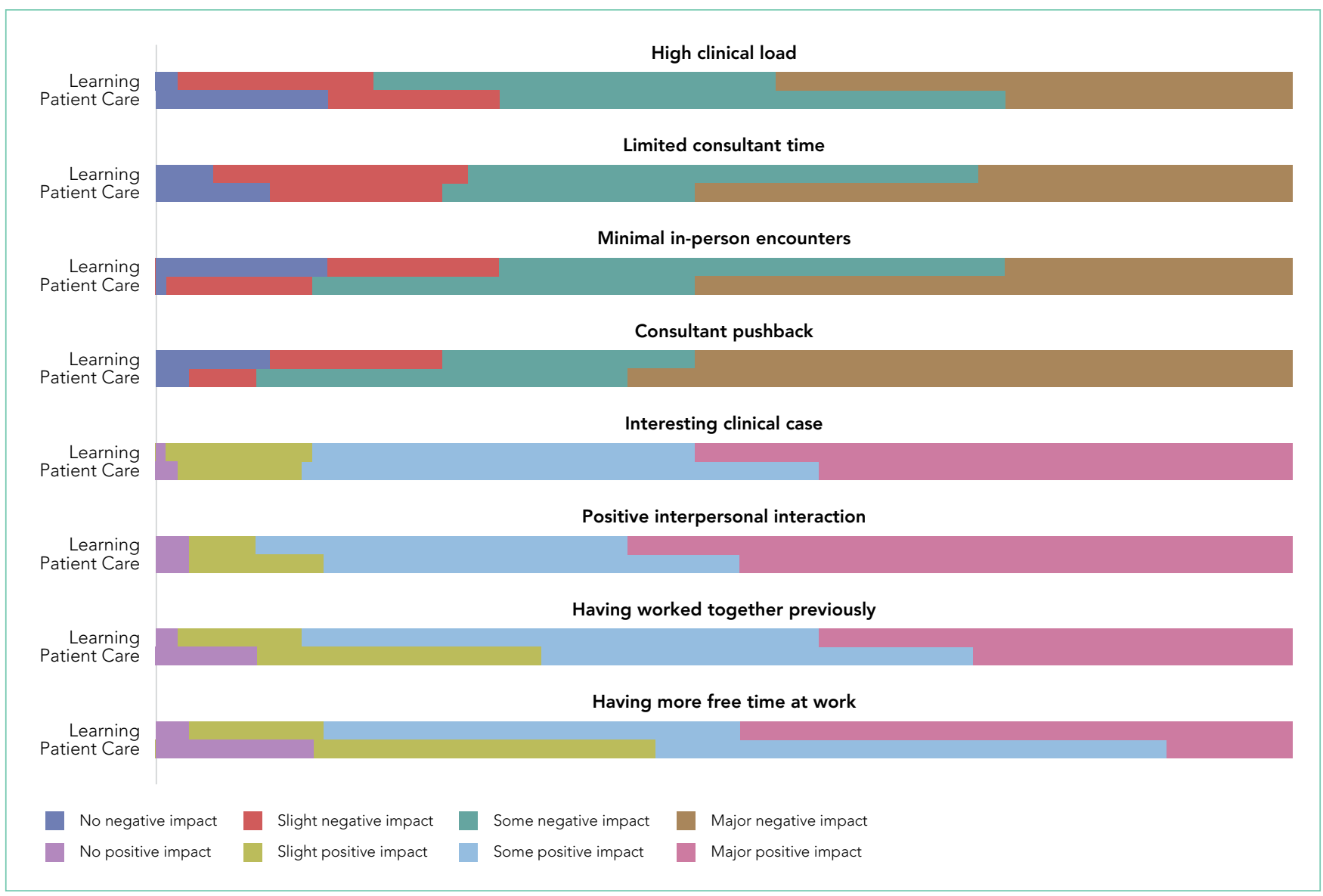

FIG 1. Barriers to and facilitating factors of patient care and hospitalist learning.

on the interaction between hospitalists and internal medicine subspecialty attendings and fellows, outline the hospitalist perspective on consultant interactions and identify a number of factors that are amenable to intervention. We found that hospitalists perceive the consult interaction to be important for patient care and a valuable opportunity for their own learning. In-person communication was seen as an important component of effective consultation but was reported to occur in a minority of consultations. We demonstrate that hospitalist-subspecialty attending consult interactions are perceived more positively than hospitalist-fellow interactions. Finally, we describe barriers and facilitating factors that may inform future interventions targeting this important interaction.

Effective communication between consultants and the primary team is critical for both patient care and teaching interactions. ${ }^{4-7}$ Pushback on consultation was reported to be the most significant barrier to hospitalist learning and had a major impact on patient care. Because hospitalists are attending physicians, we hypothesized that they may perceive pushback from fellows less frequently than residents. ${ }^{4}$ However, in our study, hospitalists reported pushback to be relatively frequent in their daily practice. Moreover, hospitalists reported a strong preference for in-person interactions with consultants, but our study demonstrated that such interactions are relatively infrequent. Researchers in studies of resident-fellow consult interactions have noted similar findings, suggesting that hospitalists and internal medicine residents face similar challenges during consultation. ${ }^{4-6}$ Hospitalists reported that positive interpersonal interactions and personal familiarity with the consultant positively affected the consult interaction. Most importantly, these effects were perceived to affect both hospitalist learning and patient care, suggesting the importance of interpersonal interactions in consultative medicine.

In an era of increasing clinical workload, the consult interaction represents an important workplace-based learning opportunity. ${ }^{4}$ Centered on a consult question, the hospitalistconsultant interaction embodies a teachable moment and can be an efficient opportunity to learn because both parties are familiar with the patient. Indeed, survey respondents reported that they frequently learned from consultation, and there was a strong preference for more teaching from consultants in this setting. However, the hospitalist-fellow consult interaction is unique because attending hospitalists are frequently communicating with fellow trainees, which could limit fellows' confidence in their role as teachers and hospitalists' perception of their role as learners. Our study identifies a number of barriers and facilitating factors (including communication, pushback, familiarity, and clinical workload) that affect the hospitalistconsultant teaching interaction and may be amenable to intervention. 
Professionalism

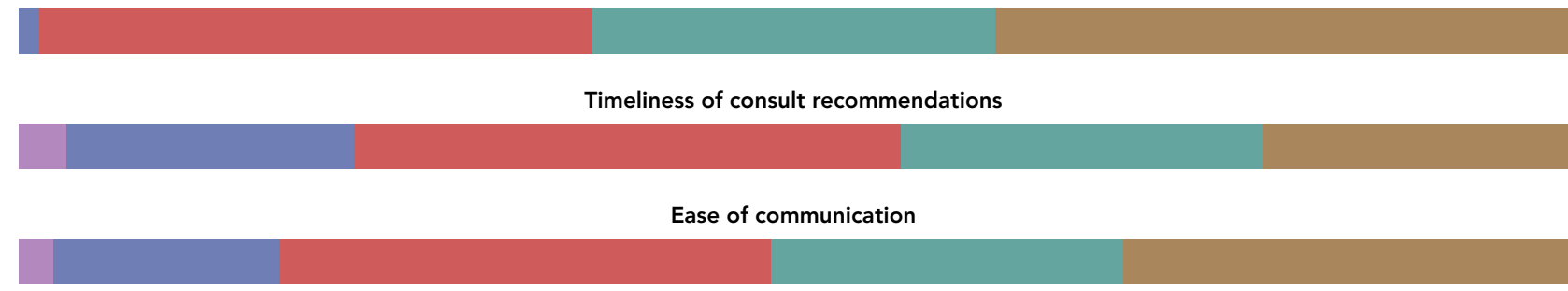

Pushback on consults

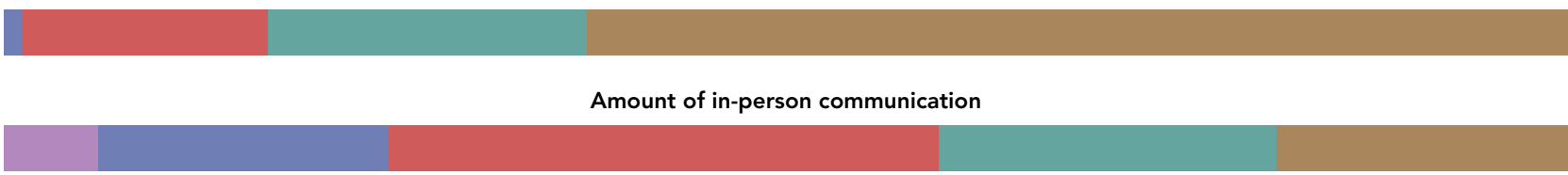

Confidence in quality of recommendations

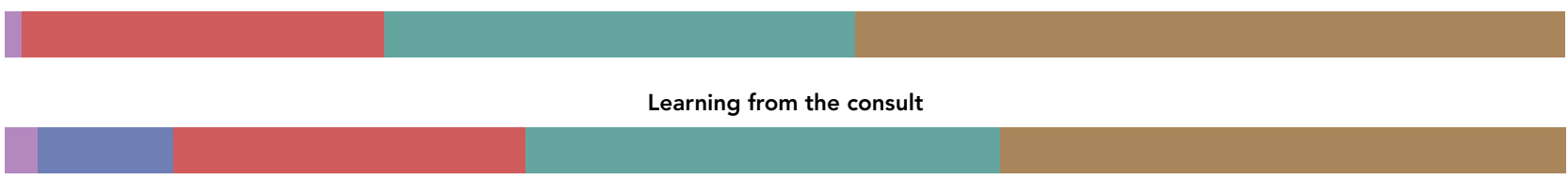

Ease of discharge planning

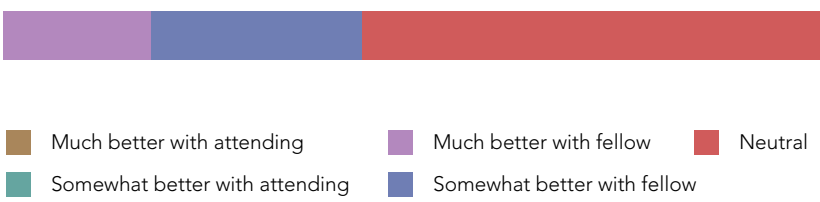

FIG 2. Hospitalist preferences with respect to consult fellows and consult attending physicians.

Hospitalists expressed a consistent preference for interacting with attending subspecialists compared to clinical fellows during consultation. Preference for interaction with attendings was strongest in the areas of pushback, confidence in recommendations, professionalism, and learning from consultation. Some of the factors that relate to consult service structure and fellow experience, such as timeliness of consultation and confidence in recommendations, may not be amenable to intervention. For instance, fellows must first see and then staff the consult with their attending prior to leaving formal recommendations, which makes their communication less timely than that of attending physicians, when they are the primary consultant. However, aspects of the hospitalist-consultant interaction (such as professionalism, ease of communication, and pushback) should not be affected by the difference in experience between fellows and attending physicians. The reasons for such perceptions deserve further exploration; however, differences in incentive structures, workload, and communication skills between fellows and attending consultants may be potential explanations.

Our findings suggest that interventions aimed at enhancing hospitalist-consultant interactions focus on enhancing direct communication and teaching while limiting the perception of pushback. A number of interventions that are primarily focused on instituting a systematic approach to requesting consultation have shown an improvement in resident and medical student consult communication ${ }^{17,18}$ as well as resident-fellow teaching interactions. ${ }^{9}$ However, it is not clear whether these interventions would be effective given that hospitalists have more experience communicating with consultants than trainees. Given the unique nature of the hospitalist-consultant interaction, multiple barriers may need to be addressed in order to have a significant impact. Efforts to increase direct communication, such as a mechanism for hospitalists to make and request in-person or direct verbal communication about a particular consultation during the consult request, can help consultants prioritize direct communication with hospitalists for specific patients. Familiarizing fellows with hospitalist workflow and the locations of hospitalist workrooms also may promote in-person communication. Fellowship training can focus on enhancing fellow teaching and communication skills, ${ }^{19-22}$ particularly as they relate to hospitalists. Fellows in particular may benefit because the hospitalist-fellow teaching interaction may be bidirectional, with hospitalists having expertise in systems practice and quality efforts that can inform fellows' practice. Furthermore, interacting with hospitalists is an opportunity for fellows to practice professional interactions, which will be critical to their careers. Increasing familiarity between fellows and hospitalists through joint events may also serve to enhance the interaction. Finally, enabling hospitalists to provide feedback to fellows stands to benefit both parties because multisource 
feedback is an important tool in assessing trainee competence and improving performance. ${ }^{23}$ However, we should note that because our study focused on hospitalist perceptions, an exploration of subspecialty fellows' and attendings' perceptions of the hospitalist-consultant interaction would provide additional, important data for shaping interventions.

Strengths of our study include the inclusion of multiple study sites, which may increase generalizability; however, our study has several limitations. The incomplete response rate reduces both generalizability and statistical power and may have created selection or nonresponder bias. However, low response rates occur commonly when surveying medical professionals, and our results are consistent with many prior hospitalist survey studies. ${ }^{24-26}$ Further, we conducted our study at a single time point; therefore, we could not evaluate the effect of fellow experience on hospitalist perceptions. However, we conducted our study in the second half of the academic year, when fellows had already gained considerable experience in the consultation setting. We did not capture participants' institutional affiliations; therefore, a subgroup analysis by institution could not be performed. Additionally, our study reflects hospitalist

\section{References}

1. Kravolec PD, Miller JA, Wellikson L, Huddleston JM. The status of hospital medicine groups in the United States. J Hosp Med.2006;1(2):75-80.

2. Cai $\mathrm{Q}$, Bruno $\mathrm{CJ}$, Hagedorn $\mathrm{CH}$, Desbiens NA. Temporal trends over ten years in formal inpatient gastroenterology consultations at an inner-city hospital. J Clin Gastroenterol. 2003;36(1):34-38.

3. Ta K, Gardner GC. Evaluation of the activity of an academic rheumatology consult service over 10 years: using data to shape curriculum. J Rheumatol. 2007:34(3):563-566

4. Miloslavsky EM, McSparron JI, Richards JB, Puig A, Sullivan AM. Teaching during consultation: factors affecting the resident-fellow teaching interaction. Med Educ. 2015:49(7):717-730

5. Chan T, Sabir K, Sanhan S, Sherbino J. Understanding the impact of residents' interpersonal relationships during emergency department referrals and consultations. J Grad Med Educ. 2013;5(4):576-581.

6. Chan T, Bakewell F, Orlich D, Sherbino J. Conflict prevention, conflict mitigation, and manifestations of conflict during emergency department consultations. Acad Emerg Med. 2014;21(3):308-313.

7. Goldman L, Lee T, Rudd P. Ten commandments for effective consultations. Arch Intern Med. 1983;143(9):1753-1755.

8. Adams T. Barriers to hospitalist fellow interactions. Med Educ. 2016;50(3):370

9. Gupta S, Alladina J, Heaton K, Miloslavsky E. A randomized trial of an intervention to improve resident-fellow teaching interaction on the wards. BMC Med Educ. 2016;16(1):276.

10. Day LW, Cello JP, Madden E, Segal M. Prospective assessment of inpatient gastrointestinal consultation requests in an academic teaching hospital. Am J Gastroenterol. 2010;105(3):484-489.

11. Kessler C, Kutka BM, Badillo C. Consultation in the emergency department: a qualitative analysis and review. J Emerg Med. 2012;42(6):704-711.

12. Salerno SM, Hurst FP, Halvorson S, Mercado DL. Principles of effective consultation: an update for the 21st-century consultant. Arch Intern Med. 2007;167(3):271-275.

13. Muzin LJ. Understanding the process of medical referral: part 1: critique of the literature. Can Fam Physician. 1991;37:2155-2161. perception rather than objectively measured communication practices between hospitalists and consultants, and it does not include the perspective of subspecialists. The specific needs of nurse practitioners and physicians' assistants, who were excluded from this study, should also be evaluated in future research. Lastly, this is a hypothesis-generating study and should be replicated in a national cohort.

\section{CONCLUSION}

The hospitalists represented in our sample population perceived the consult interaction to be important for patient care and a valuable opportunity for their own learning. Participants expressed that they would like to increase direct communication with consultants and enhance consultant-hospitalist teaching interactions. Multiple barriers to effective hospitalistconsultant interactions (including communication, pushback, and hospitalist-consultant familiarity) are amenable to intervention.

Disclosure: The authors have no financial disclosures or conflicts of interest

14. Muzin LJ. Understanding the process of medical referral: part 5: communication. Can Fam Physician. 1992;38:301-307.

15. Wadhwa A, Lingard L. A qualitative study examining tensions in interdoctor telephone consultations. Med Educ. 2006;40(8):759-767.

16. Grant IN, Dixon AS. "Thank you for seeing this patient": studying the quality of communication between physicians. Can Fam Physician. 1987;33:605-611.

17. Kessler CS, Afshar Y, Sardar G, Yudkowsky R, Ankel F, Schwartz A. A prospective, randomized, controlled study demonstrating a novel, effective model of transfer of care between physicians: the $5 \mathrm{Cs}$ of consultation. Acad Emerg Med. 2012;19(8):968-974.

18. Podolsky A, Stern DTP. The courteous consult: a CONSULT card and training to improve resident consults. J Grad Med Educ. 2015;7(1):113-117.

19. Tofil NM, Peterson DT, Harrington KF, et al. A novel iterative-learner simulation model: fellows as teachers. J. Grad. Med. Educ. 2014;6(1):127-132.

20. Kempainen RR, Hallstrand TS, Culver BH, Tonelli MR. Fellows as teachers: the teacher-assistant experience during pulmonary subspecialty training. Chest. 2005;128(1):401-406

21. Backes $\mathrm{CH}$, Reber KM, Trittmann JK, et al. Fellows as teachers: a model to enhance pediatric resident education. Med. Educ. Online. 2011;16:7205.

22. Miloslavsky EM, Degnan K, McNeill J, McSparron JI. Use of Fellow as Clinical Teacher (FACT) Curriculum for Teaching During Consultation: Effect on Subspecialty Fellow Teaching Skills. J Grad Med Educ. 2017;9(3):345-350

23. Donnon T, Al Ansari A, Al Alawi S, Violato C. The reliability, validity, and feasibility of multisource feedback physician assessment: a systematic review. Acad. Med. 2014;89(3):511-516.

24. Monash B, Najafi N, Mourad M, et al. Standardized attending rounds to improve the patient experience: A pragmatic cluster randomized controlled trial. J Hosp Med. 2017;12(3):143-149.

25. Allen-Dicker J, Auerbach A, Herzig SJ. Perceived safety and value of inpatient "very important person" services. J Hosp Med. 2017;12(3):177-179.

26. Do D, Munchhof AM, Terry C, Emmett T, Kara A. Research and publication trends in hospital medicine. J Hosp Med. 2014;9(3):148-154. 